\title{
TWO-DIMENSIONAL CELLULAR BURNER-STABILIZED FLAMES
}

\author{
By \\ R. KUSKE AND B. J. MATKOWSKY \\ Northwestern University, Evanston, Illinois
}

\begin{abstract}
We consider the behavior of a premixed flame anchored on a flat burner. For Lewis numbers $L<L^{*}<1$, one-dimensional stationary spatially periodic solutions corresponding to stationary one-dimensional cellular flames (rolls) bifurcate from the basic solution which corresponds to a steady planar flame. We derive and analyze an equation for the evolution of the amplitude of the roll solution just beyond the critical Lewis number $L^{*}$. That is, we consider the case of supercritical bifurcation $\left(L<L^{*}\right)$ and determine the ranges of wave numbers of perturbations corresponding to both the Eckhaus instability (to longitudinal perturbations) and the zigzag instability (to transverse perturbations) of the bifurcating solution. We determine these ranges in terms of the flow rate $m \in(0,1)$ and the scaled heat loss to the burner $K>2 / e$. For wave numbers $k<0.25$ we find that the zigzag instability occurs for all allowed values of $K$ and for $m$ bounded away from 1 and 0 . As $k$ increases, the range of values of $m$ and $K$ for which this instability occurs decreases. For $k \geq 0.4$ the zigzag instability no longer occurs for any allowed value of $m$ and $K$. For each value of $L$ there is a minimum value $m=m_{*}(L)$ above which the Eckhaus instability does not occur. As $L$ approaches $L^{*}, m_{*}(L)$ increases.
\end{abstract}

1. Introduction. Cellular patterns and their stability have been studied in the context of a wide variety of phenomena ranging from fluid dynamics to biology [9]. For example, in many applications one-dimensional stationary cellular patterns, or rolls, are observed. Two types of long wavelength instability which occur in these patterns are instability to longitudinal perturbations-the Eckhaus instability, and instability to transverse perturbations - the zigzag instability. The Eckhaus instability for cellular flames was analyzed using a two-dimensional model in [2], in which the flame is treated as one dimensional, that is, as a curve. The zigzag instability, associated with the rotational invariance of the rolls, leads to the undulation of the rolls along their axis, which results in a two-dimensional pattern. This modulation can be thought of as the system shifting the length of the wave vector closer to its optimal value

Received May 3, 1992.

The first author was supported by an NSF Graduate Fellowship and a Sloan Foundation Dissertation Fellowship. The second author was supported in part by DOE Grant DE-FG02-87ER25027.

(C)1994 Brown University 


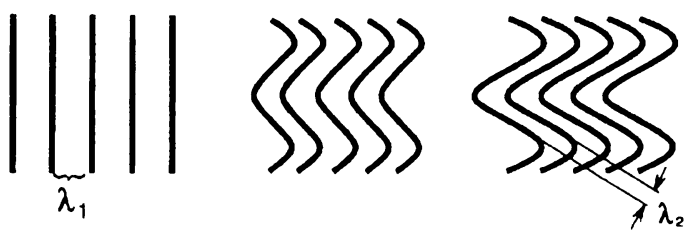

FIG. 1. The zigzag instability

$k_{c}$ by decreasing the wavelength of the rolls [10]. As can be seen from Fig. 1, the wavelength $\lambda_{2}$ of the two-dimensional pattern is smaller than $\lambda_{1}$, the wavelength of the rolls.

One model of flames anchored on a flat burner is discussed in [1] which, following [4], models the burner as a heat sink. Another model is described in [5] and [6]. The stability of a steady planar flame to one-dimensional disturbances was examined in [1] and in [8] for the model described in [5] and [6]. The behavior of two-dimensional polyhedral burner-stabilized flames was considered in [7], where instabilities arising from the interaction of two discrete modes in an effective one-dimensional burner were examined, and in [3], in which the interaction of two discrete modes in a cylindrical burner was considered. In the two-dimensional burner model used in [2], stationary cellular flames which bifurcate from the steady planar flame at a critical Lewis number $L=L^{*}<1$ were described in the context of a Ginzburg-Landau equation which was derived.

In this paper we employ a three-dimensional model of burner-stabilized flames, in which the flame is treated as a surface, to study the behavior of cellular flames arising as a bifurcation from planar flames and their stability. We derive an equation for the evolution of the amplitudes of a continuous band of unstable modes centered about the most unstable mode in the linear stability analysis of the planar flame. Onedimensional cellular solutions (rolls), which bifurcate from the basic solution for $L<$ $L^{*}$, are obtained and their stability determined by analyzing the evolution equation. We determine the stability of the cellular flame to both longitudinal and transverse perturbations, thus determining the ranges of wave numbers that correspond to the Eckhaus and zigzag instabilities, respectively. The stability regions for the wave number are determined in terms of the physical parameters of the model, the flow rate $m \in(0,1)$, and the scaled heat loss to the burner $K>2 / e$. We graph the Eckhaus and zigzag stability boundaries in the $(m, K)$-plane, indicating the regions of stability of the stationary cellular solutions that bifurcate supercritically. For wave numbers $k<0.25$ we find that the zigzag instability occurs for all regions of the $(m, K)$-plane, except for narrow bands corresponding to values of $m$ near 1 and 0 . As $k$ increases, the range of values of $m$ and $K$ for which this instability occurs decreases. For $k \geq 0.4$ the zigzag instability no longer occurs for any allowed values of $m$ and $K$. For each value of $L<L^{*}$ we find a minimum value $m=m_{*}(L)$ above which the Eckhaus instability does not occur. As $L$ decreases so does $m_{*}(L)$, so that the region of the $(m, K)$-plane for which the Eckhaus instability occurs decreases with decreasing Lewis number.

2. Formulation. The model we employ is a straightforward extension to three dimensions of that used in [1] and [2]. In this approach the burner is modeled 
as a heat sink [4], with assumptions of weak thermal expansion of the gas, large activation energy, and Lewis number close to unity. We also assume that there is a single deficient component of the reaction so that only its mass fraction evolves, while the mass fractions of all other components are so abundant that they can be considered to be constant. Moreover, the reaction is modeled by one-step Arrhenius kinetics, and the temperature of the burner is taken to be equal to that of the fresh mixture for simplicity.

We employ nondimensional cartesian coordinates $\left(x_{1}, x_{2}, x_{3}\right)$, with the burner located at $x_{1}=0$. We seek perturbation expansions of the nondimensional temperature $T\left(t, x_{1}, x_{2}, x_{3}\right)$ and mass fraction $Y\left(t, x_{1}, x_{2}, x_{3}\right)$ of the deficient component as

$$
\begin{aligned}
& T=\frac{\widetilde{T}-\widetilde{T}_{u}}{\widetilde{T}_{a}-\widetilde{T}_{u}} \sim \Theta+\frac{T_{1}}{Z}+\frac{T_{2}}{Z^{2}}+\cdots, \\
& Y=\frac{\tilde{Y}_{Y}}{\widetilde{Y}_{u}} \sim Y_{0}+\frac{Y_{1}}{Z}+\frac{Y_{2}}{Z^{2}}+\cdots
\end{aligned}
$$

where $\tilde{Y}_{u}$ is the dimensional mass fraction of the fresh mixture,

$$
Z=\frac{\widetilde{E}}{\widetilde{R} \widetilde{T}_{a}}\left(1-\frac{\widetilde{T}_{u}}{\widetilde{T}_{a}}\right) \gg 1
$$

is a nondimensional quantity termed the Zeldovich number, $\widetilde{E}$ is the dimensional activation energy, $\widetilde{T}_{u}$ and $\widetilde{T}_{a}$ are the dimensional fresh mixture and adiabatic flame temperatures, respectively, and $\widetilde{R}$ is the gas constant. Since activation energies in combustion are typically large, the reaction zone is a narrow region of width $O(1 / Z)$. As $Z \rightarrow \infty$, this zone shrinks to a front whose location is given by

$$
x_{1}=\Phi\left(t, x_{2}, x_{3}\right) \text {. }
$$

The Lewis number $L$, which is the ratio of thermal to mass diffusivities, and the heat loss coefficients $H$ are scaled as

$$
L=1+\frac{\beta}{Z}, \quad H=\frac{K}{Z} .
$$

Assuming that the reaction goes to completion at the flame front, we set $Y \equiv 0$ behind the flame.

Employing a coordinate system that moves with the flame front

$$
x=x_{1}-\Phi\left(t, x_{2}, x_{3}\right), \quad y=x_{2}, \quad z=x_{3},
$$

the model is given by

$$
\begin{aligned}
\boldsymbol{\Theta}+Y_{0} & \equiv 1 \\
\frac{\partial \boldsymbol{\Theta}}{\partial t}+\left(m-\frac{\partial \Phi}{\partial t}\right) \frac{\partial \boldsymbol{\Theta}}{\partial x} & =\Delta \boldsymbol{\Theta}+\left\{1+\left(\frac{\partial \Phi}{\partial y}\right)^{2}+\left(\frac{\partial \Phi}{\partial z}\right)^{2}\right\}^{1 / 2} \exp \left(\frac{S}{2}\right) \delta(x) \\
\frac{\partial S}{\partial t}+\left(m-\frac{\partial \Phi}{\partial t}\right) \frac{\partial S}{\partial x} & =\Delta S+\beta \Delta \Theta-K \Theta \delta(x+\Phi)
\end{aligned}
$$


with boundary conditions

$$
\begin{aligned}
& \boldsymbol{\Theta} \equiv 1 \quad \text { for } x>0, \quad \frac{\partial S}{\partial x} \rightarrow 0 \quad \text { as } x \rightarrow+\infty ; \\
& \boldsymbol{\Theta} \rightarrow 0, S \rightarrow 0 \text { as } x \rightarrow-\infty \text {; } \\
& |\Theta|<\infty,|S|<\infty \text { as } y, z \rightarrow \pm \infty \text {. }
\end{aligned}
$$

In (2.9) the parameter $m$ is the flow rate of the fuel, $S$ is defined as

$$
S \equiv T_{1}+Y_{1},
$$

and $\delta$ is the Dirac delta function. The Laplacian in the moving coordinate system is given by

$$
\begin{aligned}
\Delta= & \left\{1+\left(\frac{\partial \Phi}{\partial y}\right)^{2}+\left(\frac{\partial \Phi}{\partial z}\right)^{2}\right\} \frac{\partial^{2}}{\partial x^{2}}+\frac{\partial^{2}}{\partial y^{2}}+\frac{\partial^{2}}{\partial z^{2}} \\
& -\left\{\frac{\partial^{2} \Phi}{\partial y^{2}}+\frac{\partial^{2} \Phi}{\partial z^{2}}\right\} \frac{\partial}{\partial x}-2 \frac{\partial \Phi}{\partial y} \frac{\partial^{2}}{\partial x \partial y}-2 \frac{\partial \Phi}{\partial z} \frac{\partial^{2}}{\partial x \partial z} .
\end{aligned}
$$

3. The basic solution and its linear stability. A steady solution of (2.9)-(2.12), termed the basic solution, is given by

$$
\begin{aligned}
\Theta_{0}(x) & = \begin{cases}1, & x>0, \\
e^{m x}, & x<0 ;\end{cases} \\
S_{0}(x) & = \begin{cases}B, & x>0, \\
B-\beta m x e^{m x}, & -h<x<0, \\
B e^{m(x+h)}-\beta m x e^{m x}, & x<-h ;\end{cases} \\
\Phi_{0} & =h ;
\end{aligned}
$$

where

$$
B=2 \ln (m), \quad h=\frac{1}{m} \ln \left(\frac{-K}{B m}\right) .
$$

The basic solution (3.1)-(3.3) represents a stationary planar flame located at the standoff distance $x_{1}=h$. The $O(1 / Z)$ correction to the flame temperature, $B$, and the stand-off distance $h$ are given by (3.4). As shown in Fig. 2, $h$ is a $U$-shaped function of $m$, whose minimum approaches zero as $K$ approaches $2 / e$. We note that the basic solution becomes unbounded as $m \rightarrow 0$. Hence, only values of $m$ bounded away from zero are considered. In addition, we consider values of the heat loss parameter $K>2 / e[1]$ in order to avoid flashback $(h<0)$ for certain values of $m$. The behavior of this basic solution is discussed further in [1].

To determine the linear stability of the basic solution we introduce the perturbations

$$
\begin{gathered}
\phi=\boldsymbol{\Phi}-h, \\
w=\boldsymbol{\Theta}-\boldsymbol{\Theta}_{0}(x)-\phi \frac{d \boldsymbol{\Theta}_{0}}{d x}, \\
v=S-S_{0}(x)-\phi \frac{d S_{0}}{d x} .
\end{gathered}
$$




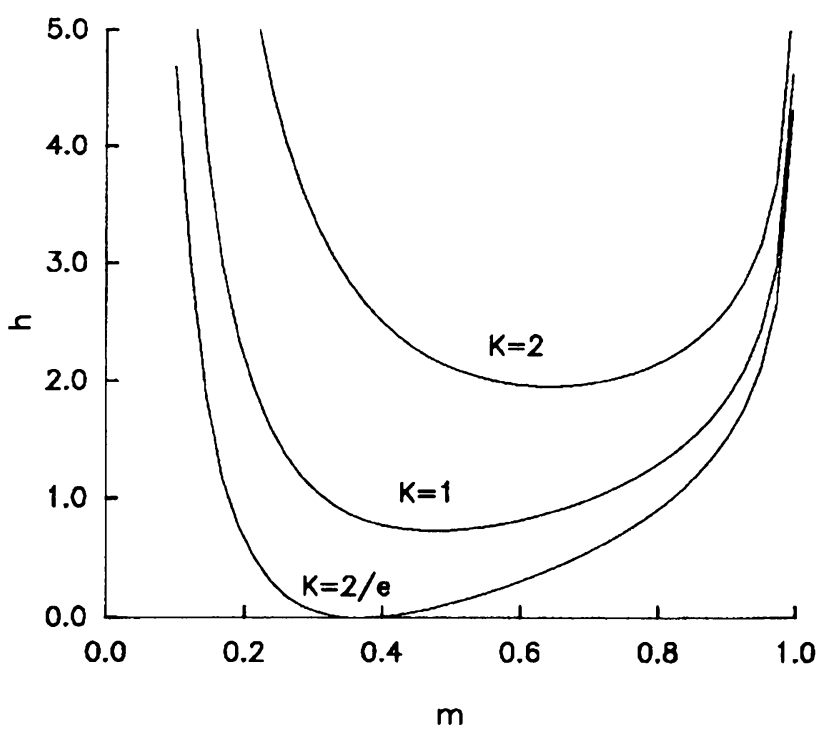

FIG. 2. Graph of $h$ vs. $m$ for $K=2 / e, 1,2$

The linear problem for these perturbations is obtained by substituting (3.7) into (2.9)-(2.12) and linearizing about $\phi=w=v=0$,

$$
\begin{aligned}
\frac{\partial w}{\partial t}+m \frac{\partial w}{\partial x}-\nabla^{2} w & =0, & x \neq 0,-h, \\
\frac{\partial v}{\partial t}+m \frac{\partial v}{\partial x}-\nabla^{2} v-\beta \nabla^{2} w & =0, & x \neq 0,-h,
\end{aligned}
$$

where$$
\nabla^{2}=\frac{\partial^{2}}{\partial x^{2}}+\frac{\partial^{2}}{\partial y^{2}}+\frac{\partial^{2}}{\partial z^{2}}
$$

with jump conditions

$$
\begin{aligned}
& \phi=\frac{1}{m}[w]_{0}, \\
& {[v]_{0}+\beta[w]_{0}=0 } \\
& {\left[\frac{\partial w}{\partial x}\right]_{0}-m[w]_{0}+\frac{m}{2} v\left(t, 0^{+}, y, z\right)=0 } \\
& {\left[\frac{\partial v}{\partial x}\right]_{0}+\beta\left[\frac{\partial w}{\partial x}\right]_{0}+\beta m[w]_{0} }=0 \\
& {[v]_{-h} }=0 \\
& {\left[\frac{\partial v}{\partial x}\right]_{-h}-K w(t,-h, y, z) }=0
\end{aligned}
$$

where $[f]_{a}$ denotes the jump in $f$ across $x=a$,

$$
[f]_{a}=f\left(t, a^{+}, y, z\right)-f\left(t, a^{-}, y, z\right),
$$


and boundary conditions

$$
\begin{gathered}
w \equiv 0 \text { for } x>0, \quad \frac{\partial v}{\partial x} \rightarrow 0 \quad \text { as } x \rightarrow+\infty ; \\
w \rightarrow 0, v \rightarrow 0 \quad \text { as } x \rightarrow-\infty \\
|w|<\infty,|v|<\infty \quad \text { as } y, z \rightarrow \pm \infty .
\end{gathered}
$$

We find that (3.8)-(3.19) has solutions of the form

$$
\left(\begin{array}{l}
w \\
v
\end{array}\right)=R e^{\omega t+i k_{1} y+i k_{2} z}\left(\begin{array}{l}
W \\
V
\end{array}\right)+\text { c.c. }
$$

where $k_{1}^{2}+k_{2}^{2}=k^{2}, R$ is an arbitrary complex constant, c.c. denotes complex conjugate,

$$
\begin{aligned}
& W= \begin{cases}0, & x>0, \\
-e^{p x}, & x<0 ;\end{cases} \\
& V= \begin{cases}A_{2} e^{l x}, & x>0, \\
C e^{l x}+D_{1} e^{p x}+D_{2} x e^{p x}, & -h<x<0, \\
E e^{p x}+D_{2} x e^{p x}, & x<-h ;\end{cases}
\end{aligned}
$$

and

$$
\begin{aligned}
p & =\frac{1}{2}\left\{m+\sqrt{m^{2}+4 \omega+4 k^{2}}\right\}, \quad l=m-p, \\
A_{2} & =\frac{2 l}{m}, \quad C=\frac{2 l}{m}+\frac{\beta\left(l^{2}-k^{2}\right)}{(p-l)^{2}}, \\
D_{1} & =\beta+\frac{2 l}{m}-C, \quad D_{2}=\frac{\beta\left(p^{2}-k^{2}\right)}{p-l}, \quad E=D_{1}+C e^{h(p-l)} .
\end{aligned}
$$

In the following sections we consider modulations of one-dimensional roll solutions. Therefore, we consider the specific wave vector $\left(k_{1}, k_{2}\right)=(k, 0)$ so that these solutions are periodic in $y$ only. Thus a distinction is made between the $y$ - and $z$-directions, and we refer to these directions as longitudinal and transverse, respectively.

The solution (3.21) and (3.22) is nontrivial if and only if the dispersion relation

$$
2 l(l-p)^{2}+\beta m\left(l^{2}-k^{2}\right)+K m(l-p) e^{-h(p-l)}=0
$$

is satisfied. For $\mathfrak{R}(\omega)<0 \quad(>0)$ the basic solution is stable (unstable). The neutral stability boundary corresponding to $\omega=0$ is shown for selected values of $m$ and $K=1$ in Fig. 3. This boundary, given explicitly by

$$
\beta=-2-8 \frac{k^{2}}{m^{2}}+\frac{2 K \alpha e^{-h \alpha}}{m(m-\alpha)},
$$

where $\alpha^{2}=m^{2}+4 k^{2}$, delineates the region of cellular instability of the basic solution. The boundary corresponding to $\mathfrak{I}(\omega) \neq 0$ is discussed in [2]. 


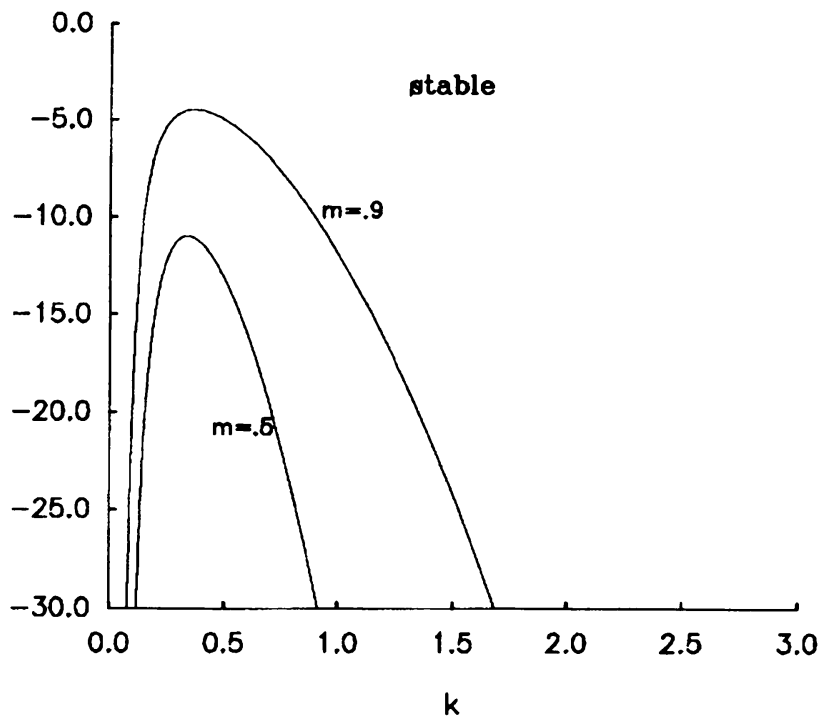

Fig. 3. Neutral stability boundary for $m=0.9$ and $m=0.5$ and $K=1$

4. Nonlinear analysis. For admissible values of the flow rate $m$, the neutral stability curve has a local maximum at $\left(\beta_{0}, k_{0}\right)$, where $k_{0} \neq 0$ corresponds to the most rapidly growing mode of the perturbation (3.20). In Figs. 4 and 5 the coordinates of the maximum are shown as functions of $m$ and $K$. From Fig. 5 we see that $k_{0}$ has a maximum value $k_{0}^{\max }$ for all $0<m<1$ and $K>2 / e$, which we refer to in Sec. 5.

We perform a local analysis in a neighborhood of the point $\left(\beta_{0}, k_{0}\right)$, seeking solutions that bifurcate from the basic solution (3.1)-(3.3). We expand in the small

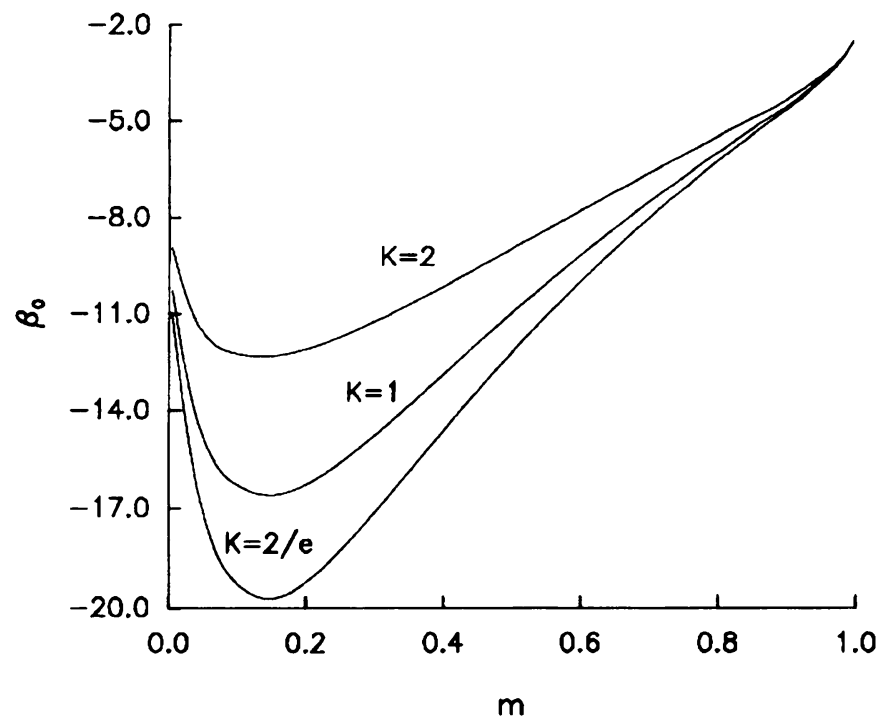

FIG. 4. Graph of $\beta_{0}$ as a function of $m$ for $K=2 / e, 1,2$. For values of $\beta<\beta_{0}$, cellular solutions exist. 


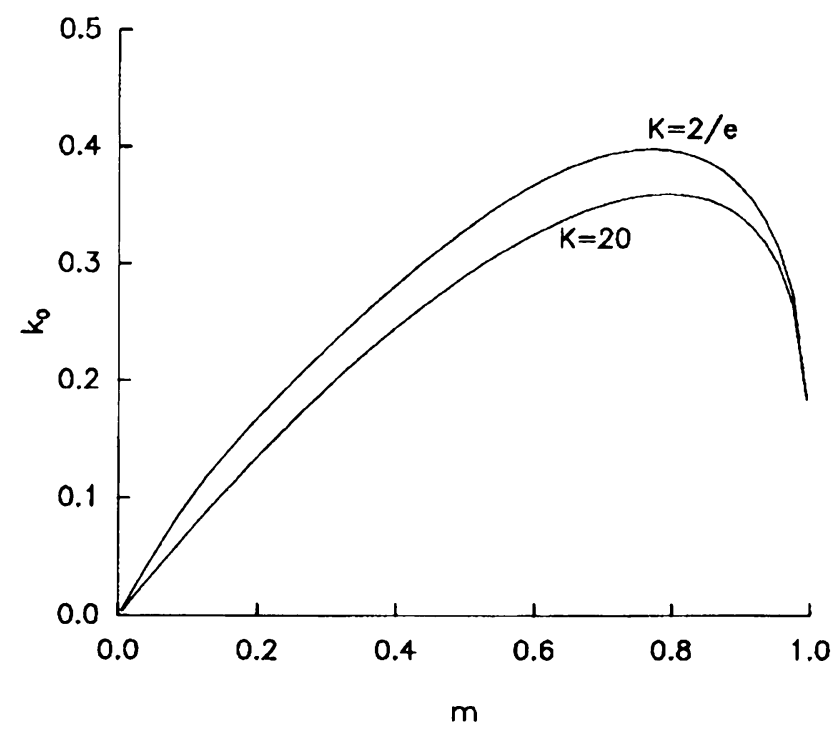

Fig. 5. Graph of $k_{0}$ as a function of $m$ for $K=2 / e$ and $K=20$

parameter $\varepsilon$ as

$$
\begin{aligned}
\beta & \sim \beta_{0}\left(1+\nu e^{2}\right)+\cdots \\
\phi & \sim \varepsilon \phi_{1}+\varepsilon^{2} \phi_{2}+\cdots \\
w & \sim \varepsilon w_{1}+\varepsilon^{2} w_{2}+\cdots
\end{aligned}
$$

and employ the scaled variables

$$
\tau=\varepsilon^{2} t, \quad \eta=\varepsilon y, \quad \zeta=\varepsilon^{1 / 2} z
$$

This choice of scalings is motivated by the fact that we are interested in the modulations of one-dimensional roll solutions directed along the $y$-direction. That is, we perturb the wave vector $\left(k_{1}, k_{2}\right)=(k, 0)$ in $(3.20)$ as $\left(k+\delta k_{y}, \delta k_{z}\right)(\delta \ll 1)$, substitute in (3.8), and balance the leading-order terms, which have coefficients $\delta k_{x}$ and $\left(\delta k_{y}\right)^{2}$.

The expansion of $\beta$ implies that the basic solution $S_{0}(x)$ is expanded as

$$
S_{0} \sim S_{00}+\varepsilon^{2} S_{02}+\cdots
$$

The perturbation $v$ is then defined as

$$
v=S-S_{0}(x)-\phi \frac{d S_{00}}{d x}
$$

and is expanded as

$$
v \sim \varepsilon v_{1}+\varepsilon^{2} v_{2}+\cdots
$$

Substituting (4.1)-(4.7) into (2.8)-(2.12) and equating like powers of $\varepsilon$ we obtain 
the sequence of equations

$$
\begin{aligned}
& \frac{\partial w_{j}}{\partial t}+m \frac{\partial w_{j}}{\partial x}-\nabla^{2} w_{j}=r_{j 1}, \quad x \neq 0,-h, \\
& \frac{\partial v_{j}}{\partial t}+m \frac{\partial v_{j}}{\partial x}-\nabla^{2} v_{j}-\beta_{0} \nabla^{2} w_{j}=r_{j 2}, \quad x \neq 0,-h \text {, }
\end{aligned}
$$

with jump conditions

$$
\begin{aligned}
& \phi_{j}=\frac{1}{m}\left[w_{j}\right]_{0}, \\
& {\left[v_{j}\right]_{0}+\beta_{0}\left[w_{j}\right]_{0}=\rho_{j 0},} \\
& {\left[\frac{\partial w_{j}}{\partial x}\right]_{0}-m\left[w_{j}\right]_{0}+\frac{m}{2} v_{j}\left(t, 0^{+}, y, z\right)=\rho_{j 1} \text {, }} \\
& {\left[\frac{\partial v_{j}}{\partial x}\right]_{0}+\beta_{0}\left[\frac{\partial w_{j}}{\partial x}\right]_{0}+\beta_{0} m\left[w_{j}\right]_{0}=\rho_{j 2},} \\
& {\left[v_{j}\right]_{-h}=\rho_{j 3},} \\
& {\left[\frac{\partial v_{j}}{\partial x}\right]_{-h}-K w_{j}(t,-h, y, z)=\rho_{j 4}}
\end{aligned}
$$

and boundary conditions

$$
\begin{gathered}
w_{j} \equiv 0 \quad \text { for } x>0, \quad \frac{\partial v_{j}}{\partial x} \rightarrow 0 \quad \text { as } x \rightarrow+\infty ; \\
w_{j} \rightarrow 0, v_{j} \rightarrow 0 \quad \text { as } x \rightarrow-\infty \\
\left|w_{j}\right|<\infty,\left|v_{j}\right|<\infty \text { as } y, z \rightarrow \pm \infty .
\end{gathered}
$$

The nonzero inhomogeneous terms $r_{j 1}, r_{j 2}, \rho_{j 0}, \rho_{j 1}, \rho_{j 2}, \rho_{j 3}, \rho_{j 4}$ for $j=1-3$ are given in Appendix A.

For $j=1$ all the inhomogeneous terms in (4.8)-(4.18) are zero, so that for a long time the one-dimensional roll solution of the homogeneous problem is

$$
\left(\begin{array}{c}
w_{1} \\
v_{1} \\
\phi_{1}
\end{array}\right)=\left(R e^{i k_{0} y}+\bar{R} e^{-i k_{0} y}\right)\left(\begin{array}{c}
W(x) \\
V(x) \\
1 / m
\end{array}\right)
$$

where $W(x)$ and $V(x)$ are given in (3.21)-(3.22) with $\beta, k$, and $\omega$ replaced by $\beta_{0}, k_{0}$, and zero, respectively. Since we perform our analysis in the neighborhood of the local maximum $\left(\beta_{0}, k_{0}\right)$ of the neutral stability curve, all other modes decay exponentially in time. At this order the complex coefficient $R(\tau, \eta, \zeta)$ and its complex conjugate $\bar{R}$ are undetermined.

Higher-order terms in the expansion of $w, v$, and $\phi$ satisfy equations (4.8)(4.18) with $j \geq 2$, which are inhomogeneous forms of the equations with $j=1$. Solutions to these problems exist if and only if certain solvability conditions are satisfied, as given in Appendix B. The small parameter $\varepsilon$ is defined so that

$$
\left\langle\left(\begin{array}{c}
w_{j} \\
v_{j}
\end{array}\right),\left(\begin{array}{l}
w^{*} \\
v^{*}
\end{array}\right)\right\rangle_{1}=0 \quad(j>1) .
$$


Thus the homogeneous part of the solutions for $j>1$ will vanish. Said another way, $\varepsilon$ is defined so that the homogeneous parts of the solution at each order are all collected into the leading-order term. Here the quantity $\left\langle\left(\begin{array}{c}w \\ v\end{array}\right),\left(\begin{array}{c}w^{*} \\ v^{*}\end{array}\right)\right\rangle_{1}$ and the vector $\left(w^{*}, v^{*}\right)^{\mathrm{T}}$ which is the long-time solution to the problem adjoint to $(3.8)-(3.19)$ with $\beta=\beta_{0}$ are given in Appendix B. For $j=2$ the solvability condition is identically satisfied, and the solution is determined to be

$$
\begin{aligned}
\left(\begin{array}{c}
w_{2} \\
v_{2} \\
\phi_{2}
\end{array}\right)= & \left(R^{2} e^{2 i k_{0} y}+\bar{R}^{2} e^{-2 i k_{0} y}\right)\left(\begin{array}{c}
W_{21}(x) \\
V_{21}(x) \\
{\left[W_{21}\right]_{0} / m}
\end{array}\right)+R \bar{R}\left(\begin{array}{c}
W_{22}(x) \\
V_{22}(x) \\
{\left[W_{22}\right]_{0} / m}
\end{array}\right) \\
& +\left\{\left(\frac{\partial^{2} R}{\partial \zeta^{2}}+2 i k_{0} \frac{\partial R}{\partial \eta}\right) e^{i k_{0} y}+\left(\frac{\partial^{2} \bar{R}}{\partial \zeta^{2}}-2 i k_{0} \frac{\partial \bar{R}}{\partial \eta}\right) e^{-k_{0} y}\right\}\left(\begin{array}{c}
W_{23}(x) \\
V_{23}(x) \\
{\left[W_{23}\right]_{0} / m}
\end{array}\right)
\end{aligned}
$$

where $W_{2 j}$ and $V_{2 j}$ for $j=1-3$ are given in Appendix C. For $j=3$ we apply the solvability conditions to obtain the evolution equation

$$
\frac{\partial R}{\partial \tau}=a \nu R+b\left(\frac{\partial}{\partial \eta}+\frac{1}{2 i k_{0}} \frac{\partial^{2}}{\partial \zeta^{2}}\right)^{2} R+d|R|^{2} R
$$

where

$$
a=\frac{m(\alpha-m)}{2}>0, \quad \alpha^{2}=m^{2}+4 k_{0}^{2},
$$

and $b$ and $d$ are given in Appendix $\mathrm{C}$. The graphs of these coefficients are shown in Figs. 6 and 7 for particular values of $K$ and for $m \in(0,1)$. For these values of $m$ and values of $K$ such that $h>0$, the coefficients $b$ and $d$ are positive and negative, respectively. The equation (4.22) is referred to as the Newell-Whitehead-Segel equation, which was first derived in the context of the Rayleigh-Benard convection $[11,12]$.

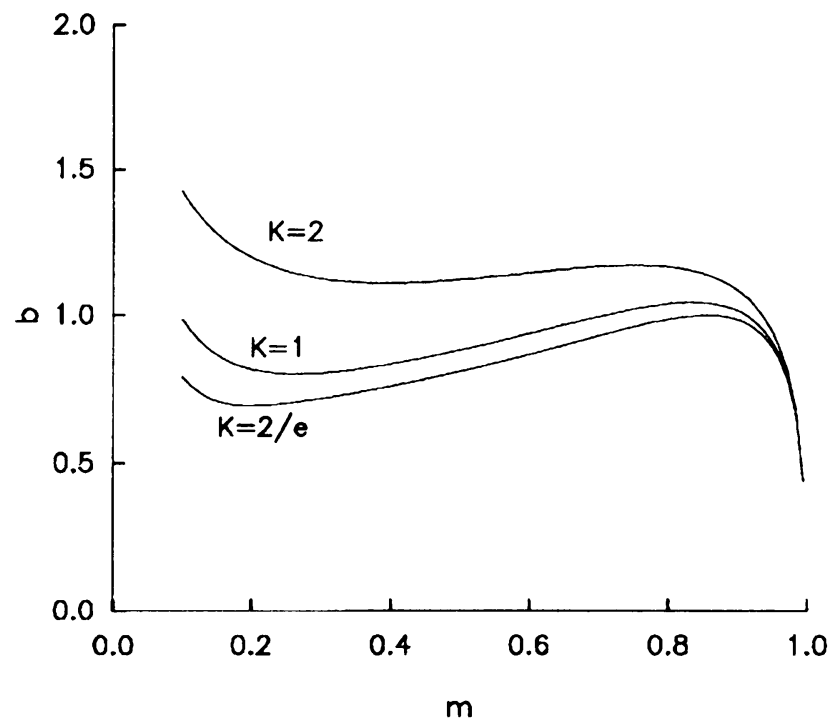

FIG. 6. Graph of $b$ vs. $m$ for $K=2 / e, 1,2$ 


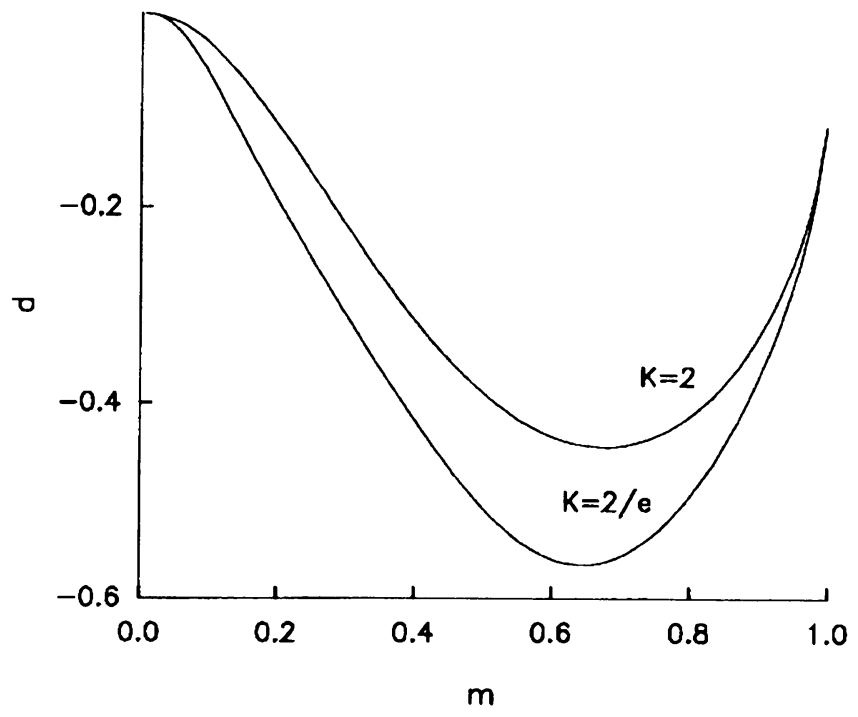

Fig. 7. Graph of $d$ vs. $m$ for $K=2 / e, 2$

5. Analysis of the amplitude equation. In this section we analyze the behavior of stationary spatially periodic solutions of $(4.22)$ given by

$$
R=r_{0} e^{i \kappa \eta}
$$

where

provided

$$
r_{0}^{2}=\frac{b \kappa^{2}-a \nu}{d}
$$

$$
\frac{b \kappa^{2}-a \nu}{d} \geq 0
$$

For the subcritical case $(\nu<0)$ this condition is never satisfied, since $d<0, a>0$, and $b>0$, while in the supercritical case $(\nu>0)(5.3)$ is satisfied by wave numbers $\kappa$ such that

$$
\kappa^{2} \leq \frac{a \nu}{b}
$$

Considering a perturbation $\mathscr{F}$ of $(5.1)$ of the form

$$
R=r_{0} e^{i \kappa \eta}+\mathscr{F}(\tau, \eta, \zeta) e^{i \kappa \eta},
$$

we determine the stability of the stationary cellular flame (5.1). The equation for $\mathscr{F}$

$$
\frac{\partial \mathscr{F}}{\partial \tau}=d r_{0}^{2}(\mathscr{F}+\bar{F})+\frac{b}{i k_{0}} \frac{\partial^{3} \mathscr{F}}{\partial \eta \partial \zeta^{2}}+\frac{b \kappa}{k_{0}} \frac{\partial^{2} \mathscr{F}}{\partial \zeta^{2}}+2 i b \kappa \frac{\partial \mathscr{F}}{\partial \eta}-\frac{b}{4 k_{0}^{2}} \frac{\partial^{4} \mathscr{F}}{\partial \zeta^{4}}+b \frac{\partial^{2} \mathscr{F}}{\partial \eta^{2}}
$$

is obtained by substituting (5.5) into (4.22) and linearizing about $\mathscr{F}=0$. Setting 
$\mathscr{F}=f+i g$, we find that $f$ and $g$ satisfy the equations

$$
\begin{aligned}
& \frac{\partial f}{\partial \tau}=2 d r_{0}^{2} f+\frac{b}{k_{0}} \frac{\partial^{3} g}{\partial \eta \partial \zeta^{2}}+\frac{b \kappa}{k_{0}} \frac{\partial^{2} f}{\partial \zeta^{2}}-2 b \kappa \frac{\partial g}{\partial \eta}-\frac{b}{4 k_{0}^{2}} \frac{\partial^{4} f}{\partial \zeta^{4}}+b \frac{\partial^{2} f}{\partial \eta^{2}}, \\
& \frac{\partial g}{\partial \tau}=-\frac{b}{k_{0}} \frac{\partial^{3} f}{\partial \eta \partial \zeta^{2}}+\frac{b \kappa}{k_{0}} \frac{\partial^{2} g}{\partial \zeta^{2}}+2 b \kappa \frac{\partial f}{\partial \eta}-\frac{b}{4 k_{0}^{2}} \frac{\partial^{4} g}{\partial \zeta^{4}}+b \frac{\partial^{2} g}{\partial \eta^{2}}
\end{aligned}
$$

which have nontrivial solutions of the form

$$
\left(\begin{array}{l}
f \\
g
\end{array}\right)=e^{\Omega t+i \mu_{2} \eta+i \mu_{3} \zeta}\left(\begin{array}{c}
\hat{f} \\
\hat{g}
\end{array}\right)
$$

for values of $\Omega$ such that

$$
\left(\Omega-2 d r_{0}^{2}+\Gamma_{1}\right)\left(\Omega+\Gamma_{1}\right)-\Gamma_{2}^{2}=0,
$$

where

$$
\begin{aligned}
& \Gamma_{1}=\frac{b \kappa}{k_{0}} \mu_{3}^{2}+\frac{b}{4 k_{0}^{2}} \mu_{3}^{4}+b \mu_{2}^{2}, \\
& \Gamma_{2}=\frac{b}{k_{0}} \mu_{2} \mu_{3}^{2}+2 b \kappa \mu_{2} .
\end{aligned}
$$

We restrict consideration to $\Omega$ real. In fact, there is a pulsating instability corresponding to $\Omega$ complex; however, it occurs in a different parameter regime and is not considered here. The solution (5.1) is stable (unstable) if $\Omega<0 \quad(>0)$. For longitudinal perturbations $\left(\mu_{2} \neq 0, \mu_{3}=0\right)$ the condition for stability is

$$
\kappa^{2}<\frac{a \nu}{3 b} \text {. }
$$

This condition, the well-known Eckhaus criterion, indicates that for the cellular flame with wave number $k$ near $k_{0}$, longitudinal perturbations of this solution decay if

$$
-\sqrt{\frac{a \nu}{3 b}}+k_{0}<k<\sqrt{\frac{a \nu}{3 b}}+k_{0} .
$$

For transverse perturbations $\left(\mu_{2}=0, \mu_{3} \neq 0\right)$ the stability condition is

$$
\kappa>0 \text {. }
$$

Stationary periodic solutions (5.1) not satisfying this criterion are said to undergo the zigzag instability. From (5.13) and (5.15) we conclude that the solution is stable to both longitudinal and transverse perturbations only if

$$
k_{0}<k<\sqrt{\frac{a \nu}{3 b}}+k_{0} .
$$

We observe that consideration of $\mu_{2}$ and $\mu_{3}$ simultaneously nonzero does not change the stability conditions. The stability condition is given for $\kappa$ in terms of the physical parameters $m, K$, and $\nu$ in Figs. 8-10. In Fig. 8 the marginal, Eckhaus, and zigzag stability boundaries are shown for $\nu=1$ and $K=1$. From Fig. 9 (see p. 678) we conclude that the stability region given in (5.16) narrows as $K$ increases. As shown in Fig. 10 (see p. 678), the width of this stability region decreases as $\nu \rightarrow 0$, that 


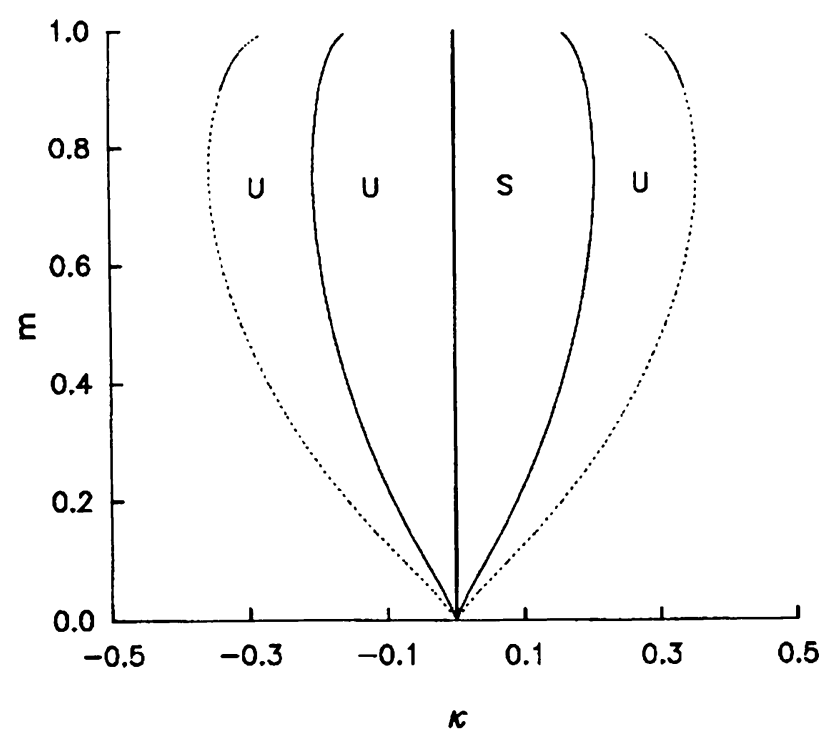

Eckhaus stability boundary neutral stability boundary

Fig. 8. Graph of the neutral, Eckhaus, and zigzag stability boundaries for $K=1$ and $\nu=1$, as given in (5.4), (5.13), and (5.15), respectively

is, as $\beta \rightarrow \beta_{0}$. This result is as expected, since $\beta_{0}$ is the maximum of the curve delineating the cellular instability region of the basic solution. The zigzag stability boundary $\left(k=k_{0}\right)$ is given in terms of $K>2 / e$ and $m$ in Fig. 11 (see p. 679). For values of $K$ exterior (interior) to the curve corresponding to $k=k^{*}$, cellular flames with wave number $k^{*}$ satisfy (violate) condition (5.15). The horizontal line corresponds to $K=2 / e$ which is the largest value of $K$ for which flashback occurs for a particular value of $m$. From the dependence of $k_{0}$ on $K$ and $m$, we conclude that for $K>2 / e$ cellular flames with wave number $k \geq k_{0}^{\max } \sim 0.4$, where $k_{0}^{\max }$ is the largest value of $k_{0}$ for any $0<m<1$ and $K>2 / e$, do not undergo the zigzag instability. As $k$ decreases from $k_{0}^{\max }$ we find that the set of values of $K$ for which the zigzag instability occurs increases in size, so that for wave numbers $k \leq 0.25$ we find that the zigzag instability occurs for all regions of the $(m, K)$-plane, except for narrow bands corresponding to values of $m$ near 1 and 0 .

In Figs. 12 and 13 (see pp. 679 and 680) we graph both the Eckhaus and zigzag stability boundaries for specific values of $k$ and $\beta$. The region corresponding to the stability of the rolls is the intersection of the region to the right of the Eckhaus stability boundary and external to the zigzag stability boundary, as described above. For fixed $k$, decreasing $\beta$ results in an increase in the size of the region of stability in the $(m, K)$-plane, as can be concluded from Fig. 10 as well. This change is due only to the increase in size of the region which is stable to longitudinal perturbations, since the zigzag stability boundary does not depend on $\beta$. In Fig. 14 (see p. 680) we plot the Eckhaus stability boundary for specific values of $k$, which indicates that the variation with $k$ of the corresponding stability region is more complex than that delineated by the zigzag stability boundary, as can be seen by the crossing of the curves. 


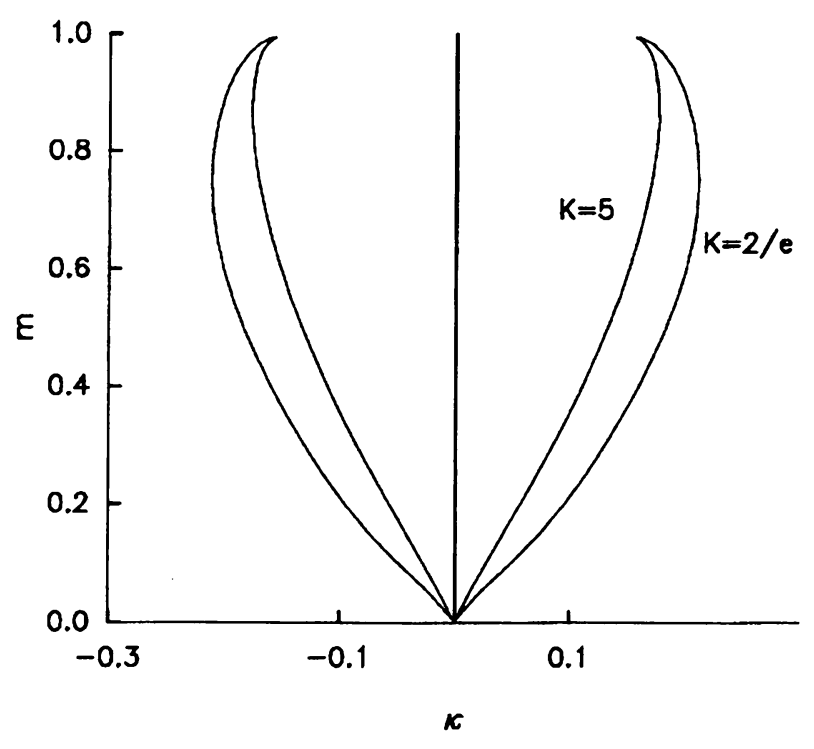

Fig. 9. The Eckhaus and zigzag stability boundaries for $K=2 / e, 5$ and $\nu=1$

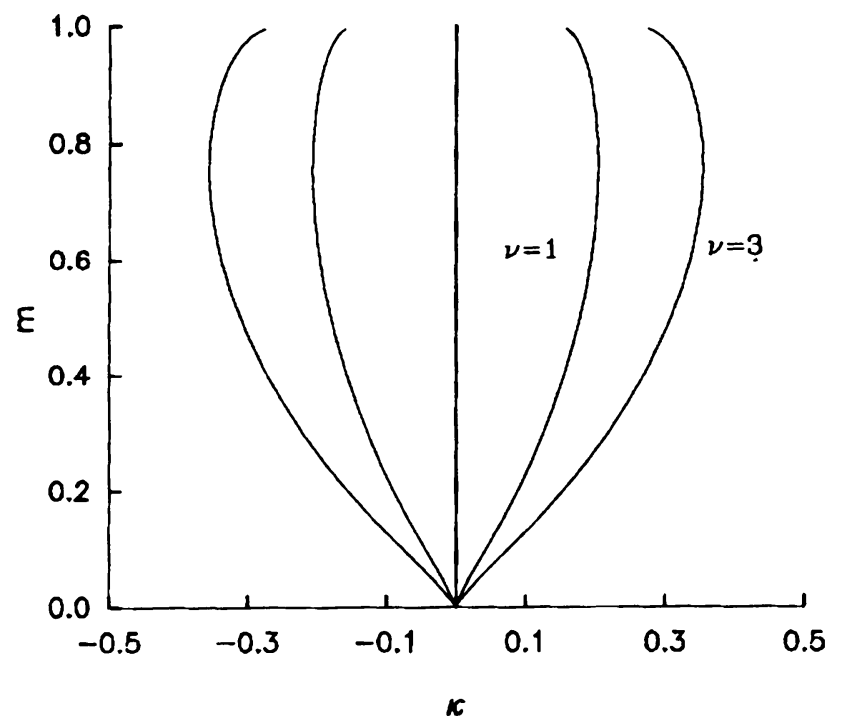

Fig. 10. The Eckhaus and zigzag stability boundaries for $K=1$ and $\nu=1,3$ 


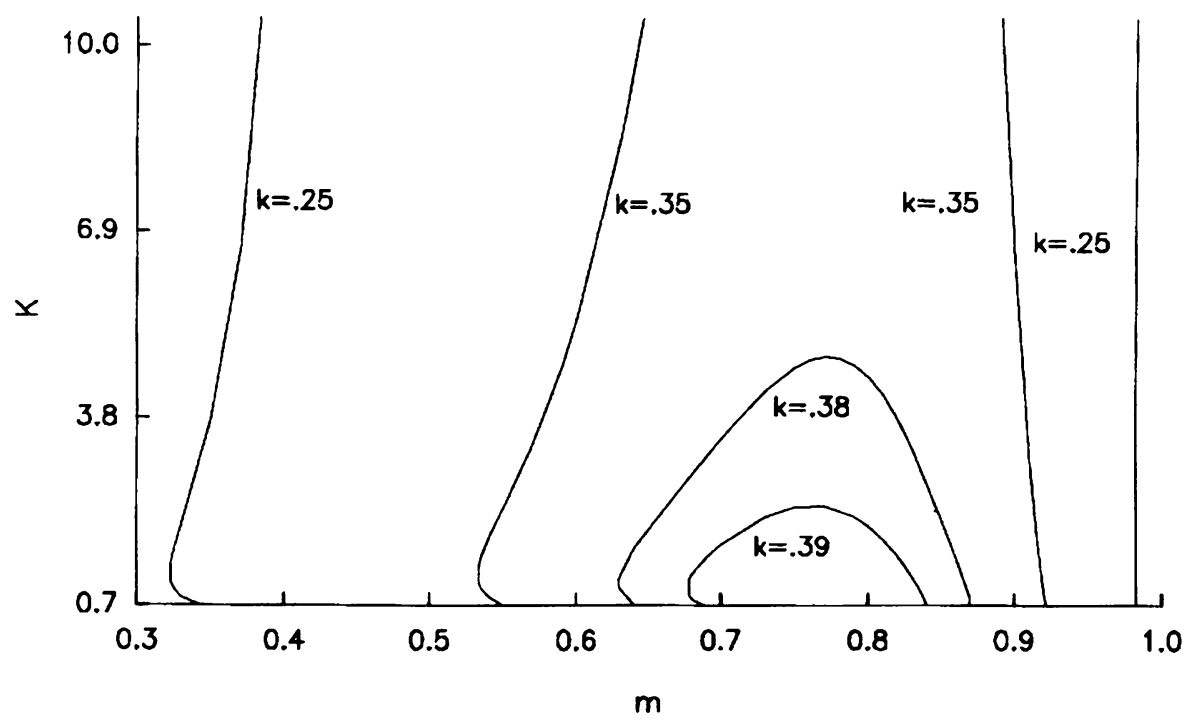

FIG. 11. Graph of the zigzag stability boundary in terms of $m$ and $K>2 / e$. For values of $K$ exterior (interior) to the curve corresponding to $k=k^{*}$ cellular flames with wave number $k^{*}$ satisfy (violate) condition (5.15).

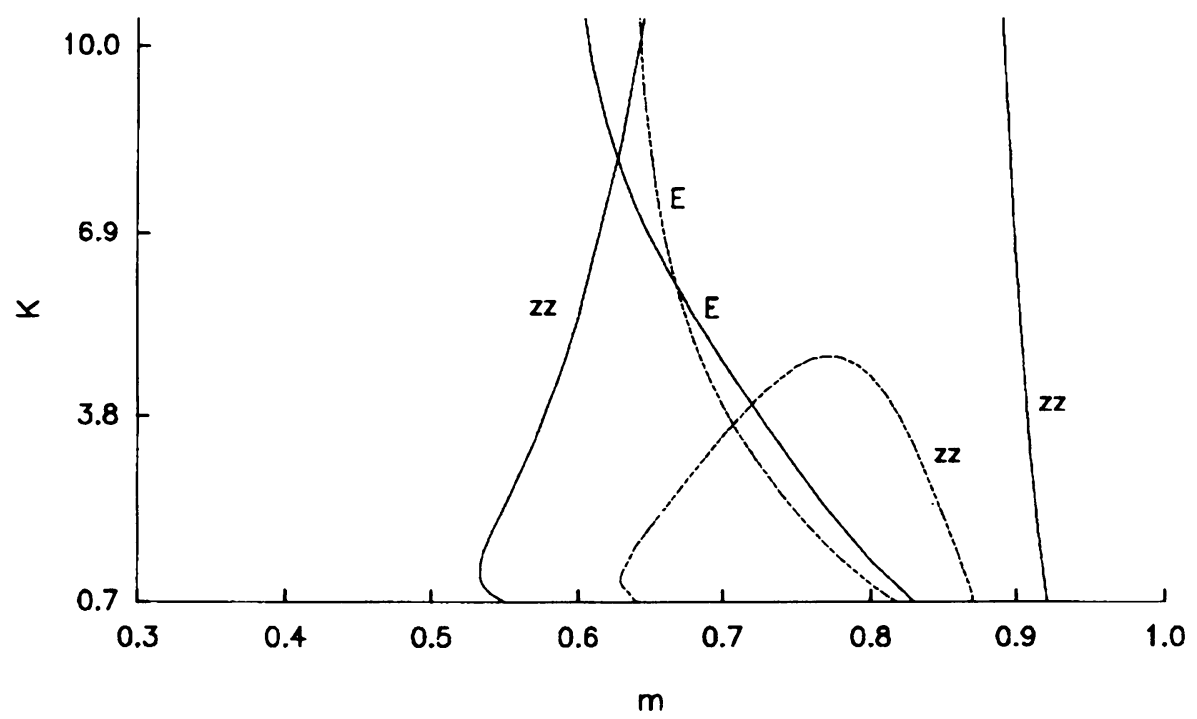

FIG. 12. Graph of the zigzag stability boundary (designated by zz) and the Eckhaus stability boundary (designated by E) for $k=0.35$, 0.38 and $\beta=-0.6$. The region of stability is the intersection of the region to the right of the Eckhaus stability boundary and the region external to the zigzag stability boundary. 


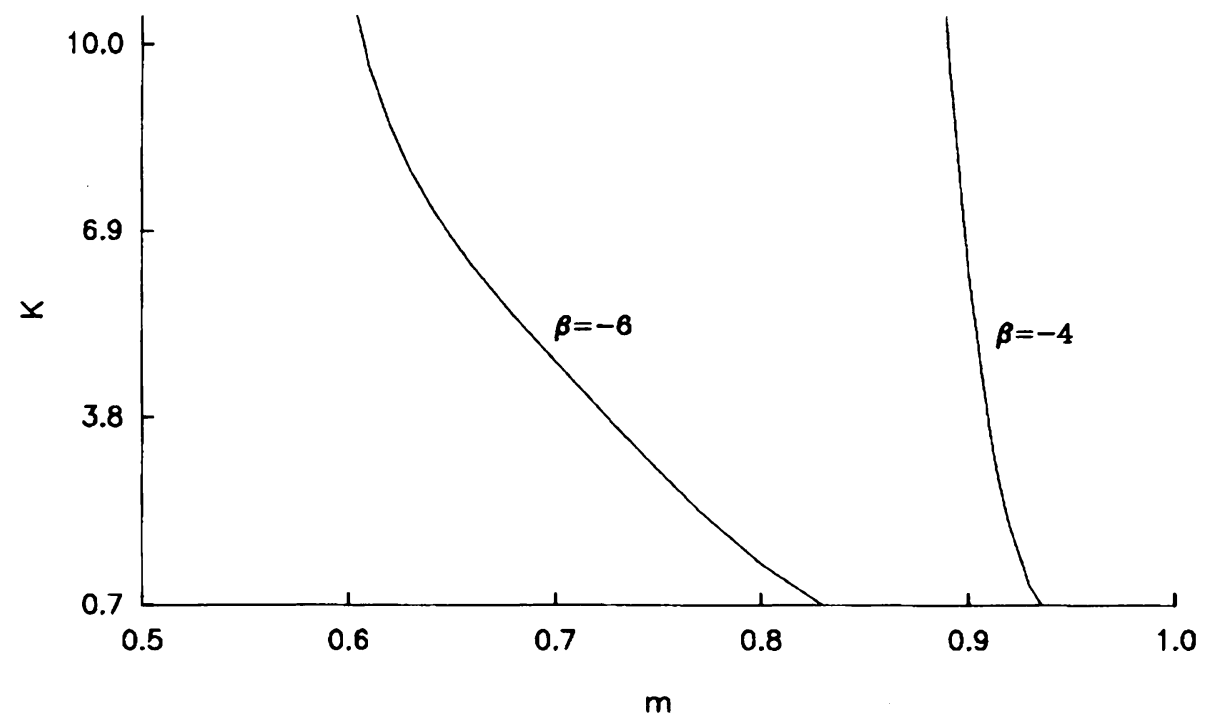

FIG. 13. Graph of the Eckhaus stability boundary for $k=0.35$ and $\beta=-6,-4$

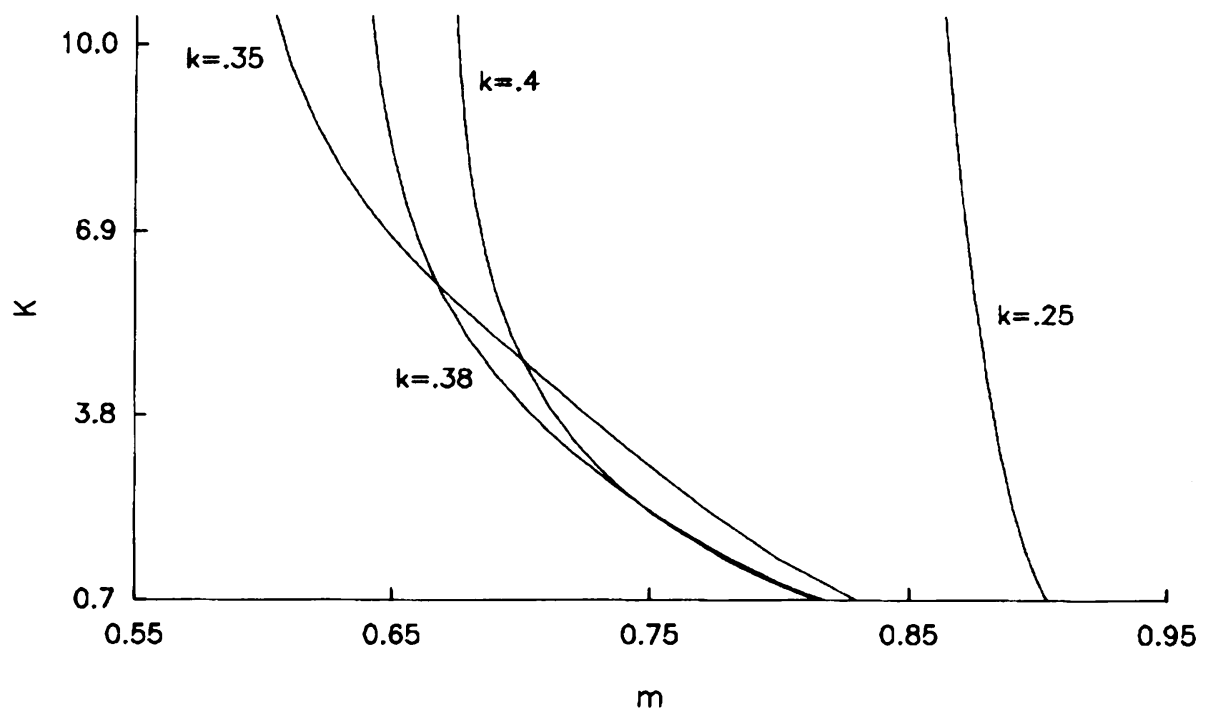

Fig. 14. Graph of the Eckhaus stability boundary for $k=0.25$, $0.35,0.38,0.4$ and $\beta=-6$

\section{Appendix A.}

The terms $r_{j 1}, r_{j 2}, \rho_{j 0}, \rho_{j 1}, \rho_{j 2}, \rho_{j 3}$, and $\rho_{j 0}$ for $j=1,2,3$, which appear on the right-hand side of the inhomogeneous problem (4.8)-(4.18) are given by

$$
r_{11}=r_{12}=\rho_{10}=\rho_{11}=\rho_{12}=\rho_{13}=\rho_{14}=0
$$




$$
\begin{aligned}
& r_{21}=-\left(\frac{\partial \phi_{1}}{\partial y}\right)^{2} \frac{d^{2} \Theta_{0}}{d x^{2}}-2 \frac{\partial \phi_{1}}{\partial y} \frac{\partial^{2} w_{1}}{\partial y \partial x}-\frac{\partial^{2} \phi_{1}}{\partial y^{2}}\left[\frac{\partial w_{1}}{\partial x}+\phi_{1} \frac{d^{2} \Theta_{0}}{d x^{2}}\right] \\
& +2 \frac{\partial^{2} w_{1}}{\partial y \partial \eta}+\frac{\partial^{2} w_{1}}{\partial \zeta^{2}} \\
& r_{22}=-\left(\frac{\partial \phi_{1}}{\partial y}\right)^{2}\left(\frac{d^{2} S_{0}^{0}}{d x^{2}}+\beta_{0} \frac{d^{2} \boldsymbol{\Theta}_{0}}{d x^{2}}\right)-2 \frac{\partial \phi_{1}}{\partial y}\left(\frac{\partial^{2} v_{1}}{\partial y \partial x}+\beta_{0} \frac{\partial^{2} w_{1}}{\partial y \partial x}\right) \\
& -\frac{\partial^{2} \phi_{1}}{\partial y^{2}}\left[\frac{\partial v_{1}}{\partial x}+\beta_{0} \frac{\partial w_{1}}{\partial x}+\phi_{1} \frac{d^{2} S_{00}}{d x^{2}}+\beta_{0} \phi_{1} \frac{d^{2} \boldsymbol{\Theta}_{0}}{d x^{2}}\right] \\
& +2\left(\frac{\partial^{2} v_{1}}{\partial y \partial \eta}+\beta_{0} \frac{\partial^{2} w_{1}}{\partial y \partial \eta}\right)+\frac{\partial^{2} v_{1}}{\partial \zeta^{2}}+\beta_{0} \frac{\partial^{2} w_{1}}{\partial \zeta^{2}} \\
& \rho_{20}=\rho_{22}=0 \text {, } \\
& \rho_{21}=\frac{m}{2}\left(\frac{\partial \phi_{1}}{\partial y}\right)^{2}-\frac{\dot{m}}{8}\left(\left.v_{1}\right|_{x=0^{+}}\right)^{2} \text {, } \\
& \rho_{23}=\frac{\phi_{1}^{2}}{2}\left[\frac{d^{2} S_{00}}{d x^{2}}\right]_{-h}+\phi_{1}\left[\frac{\partial v_{1}}{\partial x}\right]_{-h}, \\
& \rho_{24}=\frac{\phi_{1}^{2}}{2}\left[\frac{d^{3} S_{00}}{d x^{3}}\right]_{-h}+\phi_{1}\left[\frac{\partial^{2} v_{1}}{\partial x^{2}}\right]_{-h}-\left(\frac{\partial \phi_{1}}{\partial y}\right)^{2}\left[\frac{d S_{00}}{d x}\right]_{-h} \\
& -\left.K\left(\phi_{1} \frac{\partial w_{1}}{\partial x}+\frac{\phi_{1}^{2}}{2} \frac{d^{2} \Theta_{0}}{d x^{2}}\right)\right|_{-h} \text {, } \\
& r_{31}=-\frac{\partial w_{1}}{\partial \tau}+\left(\frac{\partial \phi_{1}}{\partial y}\right)^{2}\left[\frac{\partial^{2} w_{1}}{\partial x^{2}}+\phi_{1} \frac{d^{3} \boldsymbol{\Theta}_{0}}{d x^{3}}\right] \\
& -\left(2 \frac{\partial \phi_{1}}{\partial y} \frac{\partial \phi_{2}}{\partial y}+2 \frac{\partial \phi_{1}}{\partial y} \frac{\partial \phi_{1}}{\partial \eta}+\left(\frac{\partial \phi_{1}}{\partial \zeta}\right)^{2}\right) \frac{d^{2} \boldsymbol{\Theta}_{0}}{d x^{2}} \\
& -2\left(\frac{\partial \phi_{1}}{\partial y} \frac{\partial^{2} w_{2}}{\partial y \partial x}+\frac{\partial \phi_{2}}{\partial y} \frac{\partial^{2} w_{1}}{\partial y \partial x}+\frac{\partial \phi_{1}}{\partial \zeta} \frac{\partial^{2} w_{1}}{\partial \zeta \partial x}+\frac{\partial \phi_{1}}{\partial y} \frac{\partial^{2} w_{1}}{\partial \eta \partial x}+\frac{\partial \phi_{1}}{\partial \eta} \frac{\partial^{2} w_{1}}{\partial y \partial x}\right) \\
& -\left(\frac{\partial^{2} \phi_{1}}{\partial \zeta^{2}}\left[\frac{\partial w_{1}}{\partial x}+\phi_{1} \frac{d^{2} \boldsymbol{\Theta}_{0}}{d x^{2}}\right]+\frac{\partial^{2} \phi_{1}}{\partial y^{2}}\left[\frac{\partial w_{2}}{\partial x}+\phi_{2} \frac{d^{2} \boldsymbol{\Theta}_{0}}{d x^{2}}\right]\right. \\
& \left.+\frac{\partial^{2} \phi_{2}}{\partial y^{2}}\left[\frac{\partial w_{1}}{\partial x}+\phi_{1} \frac{d^{2} \boldsymbol{\Theta}_{0}}{d x^{2}}\right]+2 \frac{\partial^{2} \phi_{1}}{\partial y \partial \eta}\left[\frac{\partial w_{1}}{\partial x}+\phi_{1} \frac{d^{2} \boldsymbol{\Theta}_{0}}{d x^{2}}\right]\right) \\
& +\frac{\partial^{2} w_{2}}{\partial \zeta^{2}}+2 \frac{\partial^{2} w_{2}}{\partial y \partial \eta}+\frac{\partial^{2} w_{1}}{\partial \eta^{2}}
\end{aligned}
$$




$$
\begin{aligned}
& r_{32}=-\frac{\partial v_{1}}{\partial \tau}+\left(\frac{\partial \phi_{1}}{\partial y}\right)^{2}\left[\frac{\partial^{2} v_{1}}{\partial x^{2}}+\beta_{0} \frac{\partial^{2} w_{1}}{\partial x^{2}}+m \phi_{1} \frac{d^{2} S_{0}^{0}}{d x^{2}}\right] \\
& -\left(2 \frac{\partial \phi_{1}}{\partial y} \frac{\partial \phi_{2}}{\partial y}+2 \frac{\partial \phi_{1}}{\partial y} \frac{\partial \phi_{1}}{\partial \eta}+\left(\frac{\partial \phi_{1}}{\partial \zeta}\right)^{2}\right) m \frac{d S_{0}^{0}}{d x} \\
& -2\left(\frac{\partial \phi_{1}}{\partial y}\left[\frac{\partial^{2} v_{2}}{\partial y \partial x}+\beta_{0} \frac{\partial^{2} w_{2}}{\partial y \partial x}\right]\right. \\
& +\frac{\partial \phi_{2}}{\partial y}\left[\frac{\partial^{2} v_{1}}{\partial y \partial x}+\beta_{0} \frac{\partial^{2} w_{1}}{\partial y \partial x}\right]+\frac{\partial \phi_{1}}{\partial \zeta}\left[\frac{\partial^{2} v_{1}}{\partial \zeta \partial x}+\beta_{0} \frac{\partial^{2} w_{1}}{\partial \zeta \partial x}\right] \\
& \left.+\frac{\partial \phi_{1}}{\partial y}\left[\frac{\partial^{2} v_{1}}{\partial \eta \partial x}+\beta_{0} \frac{\partial^{2} w_{1}}{\partial \eta \partial x}\right]+\frac{\partial \phi_{1}}{\partial \eta}\left[\frac{\partial^{2} v_{1}}{\partial y \partial x}+\beta_{0} \frac{\partial^{2} w_{1}}{\partial y \partial x}\right]\right) \\
& -\left(\frac{\partial^{2} \phi_{1}}{\partial \zeta^{2}}\left[\frac{\partial v_{1}}{\partial x}+\beta_{0} \frac{\partial w_{1}}{\partial x}+m \phi_{1} \frac{d S_{0}^{0}}{d x}\right]+\frac{\partial^{2} \phi_{1}}{\partial y_{2}}\left[\frac{\partial v_{2}}{\partial x}+\beta_{0} \frac{\partial w_{2}}{\partial x}+m \phi_{2} \frac{d S_{0}^{0}}{d x}\right]\right. \\
& +\frac{\partial^{2} \phi_{2}}{\partial y^{2}}\left[\frac{\partial v_{1}}{\partial x}+\beta_{0} \frac{\partial w_{1}}{\partial x}+m \phi_{1} \frac{d S_{0}^{0}}{d x}\right] \\
& \left.+2 \frac{\partial^{2} \phi_{1}}{\partial y \partial \eta}\left[\frac{\partial v_{1}}{\partial x}+\beta_{0} \frac{\partial w_{1}}{\partial x}+m \phi_{1} \frac{d S_{0}^{0}}{d x}\right]\right) \\
& +\frac{\partial^{2} v_{2}}{\partial \zeta^{2}}+\beta_{0} \frac{\partial^{2} w_{2}}{\partial \zeta^{2}}+2 \frac{\partial^{2} v_{2}}{\partial y \partial \eta}+2 \beta_{0} \frac{\partial^{2} w_{2}}{\partial y \partial \eta}+\frac{\partial^{2} v_{1}}{\partial \eta^{2}}+\beta_{0} \frac{\partial^{2} w_{1}}{\partial \eta^{2}}+\beta_{0} \nu \nabla^{2} w_{1} \\
& -\frac{\partial^{2} \phi_{1}}{\partial y^{2}} \frac{d S_{02}}{d x}+\beta_{0} \nu \phi_{1} \frac{d^{3} \boldsymbol{\Theta}_{0}}{d x^{3}}
\end{aligned}
$$

$$
\begin{aligned}
\rho_{30}= & 0, \\
\rho_{31}= & -\frac{m}{48}\left(\left.v_{1}\right|_{x=0^{+}}\right)^{3}-\left.\frac{m}{4}\left(v_{1} v_{2}\right)\right|_{x=0^{+}}+\left.\frac{m}{4}\left(\frac{\partial \phi_{1}}{\partial y}\right)^{2} v_{1}\right|_{x=0^{+}} \\
& +m\left(\frac{\partial \phi_{1}}{\partial y} \frac{\partial \phi_{1}}{\partial \eta}+\frac{\partial \phi_{1}}{\partial y} \frac{\partial \phi_{2}}{\partial y}\right)+\frac{m}{2}\left(\frac{\partial \phi_{1}}{\partial \zeta}\right)^{2}, \\
\rho_{32}= & -\beta_{0} \nu\left[\frac{\partial w_{1}}{\partial x}-m w_{1}\right]_{0}, \\
\rho_{33}= & \phi_{1}\left[\frac{\partial v_{2}}{\partial x}\right]_{-h}+\phi_{2}\left[\frac{\partial v_{1}}{\partial x}\right]_{-h}-\frac{\phi_{1}^{2}}{2}\left[\frac{\partial^{2} v_{1}}{\partial x^{2}}\right]_{-h} \\
& +\phi_{1} \phi_{2}\left[\frac{d^{2} S_{00}}{d x^{2}}\right]_{-h}-\frac{\phi_{1}^{3}}{3}\left[\frac{d^{3} S_{00}}{d x^{3}}\right]_{-h},
\end{aligned}
$$




$$
\begin{aligned}
\rho_{34}= & \left(-2 \frac{\partial \phi_{1}}{\partial y} \frac{\partial \phi_{1}}{\partial \eta}-2 \frac{\partial \phi_{1}}{\partial y} \frac{\partial \phi_{2}}{\partial y}-\left(\frac{\partial \phi_{1}}{\partial \zeta}\right)^{2}\right)\left[\frac{d S_{00}}{d x}\right]_{-h} \\
& -\left(\frac{\partial \phi_{1}}{\partial y}\right)^{2}\left[\frac{\partial v_{1}}{\partial x}\right]_{-h}+\phi_{1}\left[\frac{\partial^{2} v_{2}}{\partial x^{2}}\right]_{-h}+\phi_{2}\left[\frac{\partial^{2} v_{1}}{\partial x^{2}}\right]_{-h}-\frac{\phi_{1}^{2}}{2}\left[\frac{\partial^{3} v_{1}}{\partial x^{3}}\right]_{-h} \\
& \left.+\phi_{1} \phi_{2}\left[\frac{d^{3} S_{00}}{d x^{3}}\right]_{-h}-\frac{\phi_{1}^{3}}{3}\left[\frac{d^{4} S_{00}}{d x^{4}}\right]_{-h} w_{1} w_{1}^{2} \frac{\partial^{2} w_{1}}{\partial w_{2}}-\phi_{1} \frac{\partial w_{2}}{\partial x}+\frac{d^{2} \Theta_{0}}{d x^{2}}+\frac{\phi_{1}^{3}}{3} \frac{d^{3} \Theta_{0}}{d x^{3}}\right)\left.\right|_{x=-h} \\
& +K(-
\end{aligned}
$$

\section{Appendix B.}

The problem adjoint to the linear homogeneous problem $(4.8)-(4.18)$ is

$$
\begin{aligned}
\frac{\partial w^{*}}{\partial t}+m \frac{\partial w^{*}}{\partial x}+\nabla^{2} w^{*}+\beta_{0} \nabla^{2} v^{*} & =0, & x \neq 0,-h, \\
\frac{\partial v^{*}}{\partial t}+m \frac{\partial v^{*}}{\partial x}+\nabla^{2} v^{*} & =0, & x \neq 0,-h,
\end{aligned}
$$

with jump conditions given by

$$
\begin{aligned}
{\left[v^{*}\right]_{0}=\left[w^{*}\right]_{0} } & =0 \\
\frac{\partial w^{*}}{\partial x} \rightarrow 0 \quad \text { as } x & \rightarrow 0^{-} \\
{\left[\frac{\partial v^{*}}{\partial x}\right]_{0}+\left.\frac{m}{2} w^{*}\right|_{x=0^{-}} } & =0 \\
{\left[v^{*}\right]_{-h}=\left[w^{*}\right]_{-h} } & =0 \\
{\left[\frac{\partial v^{*}}{\partial x}\right]_{-h} } & =0 \\
{\left[\frac{\partial w^{*}}{\partial x}\right]_{-h}-\left.K v^{*}\right|_{x=-h} } & =0
\end{aligned}
$$

and boundary conditions

$$
w^{*} \rightarrow 0, v^{*} \rightarrow 0 \text { as } x \rightarrow \pm \infty .
$$

A solution to the inhomogeneous problem exists if and only if

$$
\begin{aligned}
& \left\langle\left(\begin{array}{c}
r_{j 1} \\
r_{j 2}
\end{array}\right),\left(\begin{array}{c}
w^{*} \\
v^{*}
\end{array}\right)\right\rangle_{1} \\
& =\left\langle\left(\begin{array}{c}
\rho_{j 1} \\
\rho_{j 2}
\end{array}\right),\left(\begin{array}{c}
\left.w^{*}\right|_{x=0^{-}} \\
\left.v^{*}\right|_{x=0^{+}}
\end{array}\right)\right\rangle_{2}+\left\langle\left(\begin{array}{c}
\rho_{j 3} \\
\rho_{j 4}
\end{array}\right),\left.\left(\begin{array}{c}
-m v^{*}-\frac{\partial v^{*}}{\partial x} \\
v^{*}
\end{array}\right)\right|_{x=-h}\right\rangle_{2},
\end{aligned}
$$


with the above quantities defined by

$$
\begin{aligned}
& \left\langle\left(\begin{array}{l}
f_{1} \\
f_{2}
\end{array}\right),\left(\begin{array}{l}
g_{1} \\
g_{2}
\end{array}\right)\right\rangle_{1} \equiv \lim _{T \rightarrow \infty} \frac{1}{T} \int_{0}^{T} \int_{0}^{2 \pi / k_{0}} \int_{-\infty}^{\infty}\left(f_{1} g_{1}+f_{2} g_{2}\right) d x d y d t, \\
& \left\langle\left(\begin{array}{l}
f_{1} \\
f_{2}
\end{array}\right),\left(\begin{array}{l}
g_{1} \\
g_{2}
\end{array}\right)\right\rangle_{2} \equiv \lim _{T \rightarrow \infty} \frac{1}{T} \int_{0}^{T} \int_{0}^{2 \pi / k_{0}}\left(f_{1} g_{1}+f_{2} g_{2}\right) d y d t .
\end{aligned}
$$

For a long time the adjoint problem has the solutions

$$
\left(\begin{array}{c}
w^{*} \\
v^{*}
\end{array}\right)=e^{ \pm i k_{0} y}\left(\begin{array}{c}
W^{*} \\
V^{*}
\end{array}\right)
$$

where

$$
\begin{aligned}
& W^{*}= \begin{cases}C_{1}^{*} e^{-p x}+C_{2} x e^{-p x}, & x>0, \\
D_{1}^{*} e^{-l x}+E^{*} e^{-p x}+D_{2}^{*} x e^{-l x}, & -h<x<0, \\
F^{*} e^{-l x}+D_{2}^{*} x e^{-l x}, & x<-h,\end{cases} \\
& V^{*}= \begin{cases}A^{*} e^{-p x}, & x>0, \\
A^{*} e^{-l x}, & x<0,\end{cases}
\end{aligned}
$$

and

$$
\begin{aligned}
& A^{*}=\frac{p-l}{\beta_{0}}, \\
& C_{1}^{*}=2 A^{*} \frac{p-l}{m}, \quad C_{2}^{*}=A^{*} \beta_{0} \frac{p^{2}-k_{0}^{2}}{p-l}, \\
& D_{1}^{*}=C_{1}^{*}-E^{*}, \quad D_{2}^{*}=A^{*} \beta_{0} \frac{l^{2}-k_{0}^{2}}{l-p}, \\
& E^{*}=-C A^{*}, \quad F^{*}=D_{1}^{*}+E^{*} e^{(p-l) h},
\end{aligned}
$$

with $l, p$, and $C$ given in (3.25) with $\omega=0$ and $k=k_{0}$.

\section{Appendix C.}

The coefficients $W_{2 j}(x)$ and $V_{2 j}(x)$ for $j=1,2,3$ which are included in the definition of $w_{2}$ are given by

$$
W_{21}(x)=\frac{1}{m} \frac{d W}{d x}+\frac{1}{2 m} \frac{2 \Theta_{0}}{d x}+ \begin{cases}0, & x>0, \\ A_{21} e^{\rho x}, & x<0\end{cases}
$$




$$
V_{21}(x)=\frac{1}{m} \frac{d V}{d x}+\frac{1}{2 m^{2}} \frac{d^{2} S_{00}}{d x^{2}}+ \begin{cases}B_{21} e^{\lambda x}, & x>0, \\ C_{21} e^{\lambda x}+D_{21} e^{\rho x}+F_{21} x e^{\rho x}, & -h<x<0, \\ E_{21} e^{\rho x}+F_{21} x e^{\rho x}, & x<-h ;\end{cases}
$$

$$
\begin{aligned}
& W_{22}(x)=\frac{2}{m} \frac{d W}{d x}+\frac{A_{22}}{m} \frac{d \Theta_{0}}{d x}, \\
& V_{22}(x)=\frac{2}{m} \frac{d V}{d x}+ \begin{cases}B_{22}, & x>0 \\
C_{22}+D_{22} e^{m x}+F_{22} x e^{m x}, & -h<x<0 \\
E_{22} e^{m x}+F_{22} x e^{m x}, & x<-h\end{cases} \\
& W_{23}(x)= \begin{cases}0, & x>0 \\
A_{23} x e^{p x}, & x<0,\end{cases} \\
& V_{23}(x)= \begin{cases}B_{23} e^{l x}+C_{23} x e^{l x}, \\
D_{23} e^{l x}+E_{23} x e^{l x}+F_{23} e^{p x}+G_{23} x e^{p x}+H_{23} x^{2} e^{p x}, & -h<x<0 \\
I_{23} e^{p x}+J_{23} x e^{p x}+K_{23} x^{2} e^{p x}, & x<-h\end{cases}
\end{aligned}
$$

where

$$
\rho=\frac{1}{2}\left(m+\sqrt{m^{2}+16 k_{0}^{2}}\right), \quad \lambda=m-\rho,
$$

$$
\begin{aligned}
& F_{21}=A_{21} \beta_{0} \frac{\rho^{2}-4 k_{0}^{2}}{\lambda-\rho}, \quad F_{22}=-A_{22} \beta_{0} m \\
& A_{23}=\frac{1}{p-l}, \quad B_{23}=\frac{2}{m} A_{23}, \quad C_{23}=\frac{2 l}{m(p-l)}, \\
& D_{23}=\frac{2(1+(p-l) h) C}{(p-l)^{2}}, \quad E_{23}=\frac{C}{p-l}, \\
& F_{23}=\frac{-\beta_{0}\left(2 m^{2}-(p-l) m\right)}{(p-l)^{4}}, \quad G_{23}=\frac{\beta_{0}\left(3 m^{2}-m(p-l)-2(p-l)^{2}\right)}{2(p-l)^{3}}, \\
& H_{23}=-\frac{D_{2}}{(p-l)}, \quad I_{23}=\frac{2 K}{(p-l)^{3}}+F_{23}, \\
& J_{23}=G_{23}-\frac{K}{(p-l)^{2}}, \quad K_{23}=H_{23} .
\end{aligned}
$$


The coefficients $A_{2 j}, B_{2 j}, C_{2 j}, D_{2 j}$, and $E_{2 j}$ for $j=1,2$ are solutions of the linear equations

$$
M_{21}\left(\begin{array}{l}
A_{21} \\
B_{21} \\
C_{21} \\
D_{21} \\
E_{21}
\end{array}\right)=\left(\begin{array}{l}
s_{1} \\
s_{2} \\
s_{3} \\
s_{4} \\
s_{5}
\end{array}\right)
$$

and

$$
M_{22}\left(\begin{array}{l}
A_{22} \\
B_{22} \\
C_{22} \\
D_{22} \\
E_{22}
\end{array}\right)=\left(\begin{array}{l}
t_{1} \\
t_{2} \\
t_{3} \\
t_{4} \\
t_{5}
\end{array}\right)
$$

where the matrices $M_{21}$ and $M_{22}$ are given by

$$
M_{21}=\left(\begin{array}{ccccc}
-\beta_{0} & 1 & -1 & -1 & 0 \\
\lambda & m / 2 & 0 & 0 & 0 \\
-\beta_{0}\left(m+\rho+\frac{\rho^{2}-4 k_{0}^{2}}{\lambda-\rho}\right) & \lambda & -\lambda & -\rho & 0 \\
0 & 0 & e^{-\lambda h} & e^{-\rho h} & -e^{-\rho h} \\
-K e^{-\rho h} & 0 & \lambda e^{-\lambda h} & \rho e^{-\rho h} & -\rho e^{-\rho h}
\end{array}\right)
$$

and

$$
M_{22}=\left(\begin{array}{ccccc}
-\beta_{0} & 1 & -1 & -1 & 0 \\
0 & 1 & 0 & 0 & 0 \\
-\beta_{0} m & 0 & 0 & -m & 0 \\
0 & 0 & 1 & e^{-m h} & -e^{-m h} \\
-K e^{-m h} & 0 & 0 & m e^{-m h} & -m e^{-m h}
\end{array}\right)
$$

and the right-hand sides of (C.9) and (C.10) are

$$
\left(\begin{array}{c}
s_{1} \\
s_{2} \\
s_{3} \\
s_{4} \\
s_{5}
\end{array}\right)=\left(\begin{array}{c}
\beta_{0} / 2 \\
3 l(p-l) / 2 m \\
\beta_{0} m / 2 \\
0 \\
-B k_{0}^{2} / m
\end{array}\right), \quad\left(\begin{array}{l}
t_{1} \\
t_{2} \\
t_{3} \\
t_{4} \\
t_{5}
\end{array}\right)=\left(\begin{array}{c}
2 \beta_{0} \\
-2 l(m-2(p-l)) / m^{2} \\
2 \beta_{0} m \\
-B \\
2 B k_{0}^{2} / m
\end{array}\right) .
$$


The coefficients $b$ and $d$ in the evolution equation (4.23) are given by

$$
\begin{aligned}
b= & -4 k_{0}^{2} \frac{\alpha}{\beta_{0}}\left(\frac{-4 h(m-\alpha)}{\alpha^{2} m}+\frac{-2 m^{2}-4 \alpha^{2}+8 m \alpha}{\alpha^{3} m(m-\alpha)}-\frac{\beta_{0}\left(m^{3}-2 m^{2} \alpha\right)}{\alpha^{5}(m-\alpha)}\right), \quad(\mathrm{C} .14) \\
\frac{\beta_{0}}{\alpha} d= & \frac{2 \alpha k_{0}^{2}}{m^{2}}-\alpha \frac{-4 m^{3}+7 m^{2} \alpha-4 m \alpha^{2}+\alpha^{3}}{4 m^{3}}-9 k_{0}^{2} \alpha \frac{m^{2}-(\alpha+\delta) m+\alpha \delta}{2(\alpha+\delta) m^{3}} \\
& +\beta_{0} k_{0}^{2}\left(\frac{3(m+\delta)^{2}}{4 m(\alpha+\delta) \delta}-\frac{2 m^{2}-m \alpha+4 \alpha^{2}}{m \alpha(m+\alpha)}\right) \\
& +B\left(\frac{(m+\alpha)\left(-9 m^{2}+6 m \alpha-5 \alpha^{2}\right)}{8 m^{2}} e^{h l}-\frac{3 k_{0}^{4} \lambda e^{h \lambda}}{m^{2}(\lambda-l) \delta}+\frac{3 k_{0}^{4} l e^{l h}}{m^{2}(\lambda-l)(\rho-l)}\right. \\
& \left.\left.-A_{22}\left(\alpha B e^{2 h l}-\frac{m(m-\alpha) B}{4}\right)-\frac{5}{2} \alpha-\frac{7 \alpha^{2}}{4 m}\right)-\frac{\alpha(m-\alpha)\left(m^{2}+\alpha^{2}\right)}{4 m^{3}}\right) \\
& +A_{21}\left[3 k_{0}^{2} \frac{m^{2} \alpha+3 \delta^{2} \alpha-2 m \alpha \delta+2 m \delta^{2}-\delta^{3}-m^{2} \delta}{m^{2}\left(\alpha^{2}-\delta^{2}\right)}-\frac{\alpha(m-\alpha)(m-\delta)}{2 m^{2}}\right. \\
+\beta_{0}( & \frac{3 k_{0}^{2}(m+\delta)\left(-2 \delta \alpha m^{2}-5 \delta^{2} m^{2}-m^{2} \alpha^{2}+4 m \alpha^{2} \delta+4 m \alpha \delta^{2}-2 \delta^{3} \alpha+\delta^{2} \alpha^{2}+\delta^{4}\right)}{4 m(\alpha-\delta)(\alpha+\delta)^{2} \delta^{2}} \\
\left.+12 k_{0}^{4} \frac{3 \delta m+\alpha m-\alpha \delta+\delta^{2}}{m(\alpha+\delta)^{2} \delta^{2}}+\frac{2 k_{0}^{2}}{m+\alpha}\right)+\frac{2 k_{0}^{2} \alpha}{m^{2}} & \left.+B\left(\left(-m+\frac{\alpha^{2}}{m}\right) e^{h l}-\frac{3 k_{0}^{2} \lambda e^{2 \lambda h}}{(\lambda-l) \delta}-\alpha e^{2 h l}\right)\right]
\end{aligned}
$$

where

$$
\begin{aligned}
\delta & =\rho-\lambda, \\
A_{22} & =\frac{m^{2}+2 k_{0}^{2}}{m^{2}}-\frac{(m-\alpha)(m-2 \alpha)}{B m^{2}}, \\
A_{21} & =\frac{A_{21}^{n}}{A_{21}^{d}} \\
A_{21}^{n} & =\frac{-3(m-\alpha) \alpha}{2 m^{2}}+\frac{B k_{0}^{2} e^{\lambda h}}{\delta m}-\frac{\beta_{0} m}{4 \delta}+\frac{\beta_{0}}{4}, \\
A_{21}^{d} & =-\frac{\beta_{0}}{4}+\frac{\beta_{0} m}{2 \delta}-\frac{\beta_{0} m^{2}}{4 \delta^{2}}+\frac{4 k_{0}^{2} \beta_{0}}{\delta^{2}}+\frac{\delta}{m}-\frac{m B e^{2 \lambda h}}{\delta}-1 .
\end{aligned}
$$

In (C.14)-(C.16) the variables $p, l, \rho, \lambda, \alpha$, and $\delta$ are all evaluated at $k=k_{0}$. 


\section{REFERENCES}

[1] B. J. Matkowsky and D. Olagunju, Pulsations in a burner stabilized premixed plane flame, SIAM J. Appl. Math. 40, 551-562 (1981)

[2] D. Olagunju and B. J. Matkowsky, Burner stabilized cellular flames, SIAM J. Appl. Math. 48, 645-664 (1990)

[3] D. Olagunju and B. J. Matkowsky, Polyhedral flames, SIAM J. Appl. Math. 51, 73-89 (1991)

[4] G. F. Carrier, F. E. Fendell, and W. B. Bush, Stoichiometry and flameholder effects on a one-dimensional flame, Comb. Sci. Tech. 18, 33-46 (1978)

[5] J. D. Buckmaster and S. S. Ludford, Lectures in mathematical combustion, CBMS Regional Conference Series in Applied Mathematics, vol. 43, SIAM, Philadelphia, 1983

[6] J. D. Buckmaster and S. S. Ludford, Theory of Laminar Flames, Cambridge Univ. Press, 1982

[7] J. D. Buckmaster, Polyhedral flames-an exercise in bimodal bifurcation analysis, SIAM J. Appl. Math. 44, 40-55 (1984)

[8] S. B. Margolis, Bifurcation phenomena in burner-stabilized premixed flames, Comb. Sci. Tech. 22, 143-169 (1980)

[9] A. C. Newell, The Dynamics and Analysis of Patterns, in Lectures in the Sciences of Complexity (D. L. Stein, ed.), Addison-Wesley, New York, 1989

[10] P. Manneville, Dissipative Structures and Weak Turbulence, Academic Press, San Diego, 1990

[11] A. C. Newell and J. A. Whitehead, Finite bandwidth, finite amplitude convection, J. Fluid Mech. 38, 279-303 (1969)

[12] L. A. Segel, Distant sidewalls cause slow amplitude modulation of cellular convection, J. Fluid Mech. 38, 203-224 (1969) 